\title{
A GENERAL ISEPIPHANIC INEQUALITY
}

\author{
ERWIN LUTWAK
}

\begin{abstract}
An inequality of Petty regarding the volume of a convex body and that of the polar of its projection body is shown to lead to an inequality between the volume of a convex body and the power means of its brightness function. A special case of this power-mean inequality is the classical isepiphanic (isoperimetric) inequality. The power-mean inequality can also be used to obtain strengthened forms and extensions of some known and conjectured geometric inequalities. Affine projection measures (Quermassintegrale) are introduced.
\end{abstract}

In [12] it was shown that the Blaschke-Santaló inequality [23] leads immediately to a power-mean inequality relating the volume of a convex body and the power means of its width (function). Special cases of this power-mean inequality include the classical inequalities of Urysohn and Bieberbach.

It will be shown in the present note that an inequality of Petty [19], which we will refer to as the Petty projection inequality, leads immediately to an analogous power-mean inequality relating the volume of a convex body and the power means of its brightness (function). A special case of this power-mean inequality is the classical isepiphanic (isoperimetric) inequality. This power-mean inequality also leads to inequalities similar to some width-volume inequalities obtained by Chakerian $[6,7]$, Chakerian and Sangwine-Yager [8], and the author [15]. When combined with ot: $r$ known inequalities, this power-mean inequality can be used to obtain a strengthened form of an inequality of Knothe [11] and Chakerian [5] relating the volume of a convex body and the arithmetic mean of the volumes of its circumscribed right cylinders. Finally, it solves completely a problem posed in [14], and can be used to prove two (similar) conjectures of the author $[\mathbf{1 6}, \mathbf{2 6}]$.

The setting for this note is Euclidean $n$-dimensional space, $\mathbf{R}^{n}(n \geq 2)$. We will use the letter $K$ (possibly with subscripts) to denote a convex body (compact convex set with nonempty interior) in $\mathbf{R}^{n}$. We use $S^{n-1}$ to denote the surface and $\omega_{n}$ to denote the $n$-dimensional volume of the unit ball in $\mathbf{R}^{n}$. The letter $u$ will denote a unit vector, exclusively. For a given direction $u \in S^{n-1}$, we use $E_{u}$ to denote the hyperplane (passing through the origin) orthogonal to $u$. For a given $K$ and $u \in S^{n-1}$, we use $b_{K}(u)$ and $\sigma_{K}(u)$ to denote respectively the width and brightness of $K$ in the direction $u$; i.e., $\sigma_{K}(u)$ is the $(n-1)$-dimensional volume of the projection of $K$ onto $E_{u}$, while $b_{K}(u)$ is the 1-dimensional volume of the projection of $K$ onto the orthogonal complement of $E_{u}$. For the volume, surface area, and mean width of $K$, we write $V(K), S(K)$, and $B(K)$, respectively. The reader is referred to [3 and 9] for material relating to convex bodies.

Received by the editors January 12, 1983.

1980 Mathematics Subject Classification. Primary 52A40; Secondary 53A15, 46B20.

Key words and phrases. Convex body, projection body, projection measure (Quermassintegrale). 
For a positive continuous function $f$ defined on $S^{n-1}$ and a real number $p \neq 0$, the $p$-mean of $f, M_{p}[f]$, is defined by

$$
M_{p}[f]=\left[\frac{1}{n \omega_{n}} \int_{S^{n-1}} f^{p}(u) d S(u)\right]^{1 / p},
$$

where $d S(u)$ is the surface area element on $S^{n-1}$ at $u$. For $p=-\infty, 0$, or $\infty, M_{p}[f]$ is defined by

$$
M_{p}[f]=\lim _{s \rightarrow p} M_{s}[f] .
$$

It is well known $\left[10\right.$, p. 143] that $M_{p}[f]$ is continuous in $p$, and that

$$
M_{\infty}[f]=\max \left\{f(u) \mid u \in S^{n-1}\right\},
$$

while,

$$
M_{-\infty}[f]=\min \left\{f(u) \mid u \in S^{n-1}\right\} .
$$

If $p<q$, then we have Jensen's inequality [10, p. 144],

$$
M_{p}[f] \leq M_{q}[f],
$$

with equality if and only if $f$ is constant.

For a convex body $K$ in $\mathbf{R}^{n}$ and a point $*$ in the interior of $K$, let $K^{*}$ denote the polar reciprocal body of $K$ with respect to the unit sphere centered at $*$. The Blaschke-Santaló inequality is

$$
\operatorname{Inf} V(K) V\left(K^{*}\right) \leq \omega_{n}^{2},
$$

with equality if and only if $K$ is an ellipsoid,

where the Inf is taken over all points $*$ in the interior of $K$. The inequality is due to Blaschke [1] for $n \leq 3$ and to Santaló [23] for $n \geq 2$ (see also the comments in Schneider [25, p. 552]). From these works also follow the conditions for equality when $K$ is assumed to be sufficiently smooth. The conditions for equality for arbitrary convex bodies were recently obtained by Petty [21]. (See also Saint Raymond [22] for the case where $K$ is assumed centrally symmetric.)

In [12] it was shown that a direct consequence of (2) is

TheOREM 1. For $p>-n$ and for all convex bodies $K$ in $\mathbf{R}^{n}$

$$
\left[V(K) / \omega_{n}\right]^{1 / n} \leq M_{p}\left[b_{K} / 2\right],
$$

with equality if and only if $K$ is a ball.

If $p=-n$, the inequality remains valid; however, equality can occur if and only if $K$ is an ellipsoid. For $p<-n$, the inequality does not hold (for all $K$ ).

As noted in [12], since $M_{\infty}\left[b_{K} / 2\right]$ is one-half the diameter of $K$, the case $p=\infty$ in Theorem 1 is the Bieberbach inequality [9, p. 173], and since $M_{1}\left[b_{K} / 2\right]$ is equal to $B(K) / 2$, the case $p=1$ is the Urysohn inequality $[3$, p. 76].

Clearly, from (1), it follows that for $p<q$ we have

$$
M_{p}\left[b_{K} / 2\right] \leq M_{q}\left[b_{K} / 2\right],
$$

with equality if and only if $K$ is of constant width.

From (3), we see that, in Theorem 1, larger values of $p$ result in 'weaker' inequalities.

As will be shown presently, the Petty projection inequality leads immediately to a result analogous to Theorem 1: 
Theorem 2. For $p>-n$ and for all convex bodies $K$ in $\mathbf{R}^{n}$

$$
\begin{aligned}
& {\left[V(K) / \omega_{n}\right]^{(n-1) / n} \leq M_{p}\left[\sigma_{K} / \omega_{n-1}\right],} \\
& \text { with equality if and only if } K \text { is a ball. }
\end{aligned}
$$

For $p=-n$, the inequality remains valid; however, equality can occur if and only if $K$ is an ellipsoid. For $p<-n$, the inequality does not hold (for all $K)$.

Since the Cauchy surface area formula $[9$, p. 208] is

$$
S(K)=n \omega_{n} M_{1}\left[\sigma_{K} / \omega_{n-1}\right]
$$

the case $p=1$ in Theorem 2 is the classical isepiphanic (isoperimetric) inequality

$$
\begin{aligned}
& n \omega_{n}^{1 / n} V(K)^{(n-1) / n} \leq S(K), \\
& \text { with equality if and only if } K \text { is a ball. }
\end{aligned}
$$

From (1), it follows immediately that for $p<q$ we have

$$
M_{p}\left[\sigma_{K} / \omega_{n-1}\right] \leq M_{q}\left[\sigma_{K} / \omega_{n-1}\right]
$$

with equality if and only if $K$ is of constant brightness.

From (4), we see that, in Theorem 2, larger values of $p$ result in 'weaker' inequalities. We note that inequality (4) for the case $p=-n, q=1$ can be found in Petty [19, p. 40].

We now prove Theorem 2. For a given convex body $K$, the projection body of $K, \Pi K$ is defined [3, p. 45] (see also Bolker [2] and Schneider-Weil [26]) as the convex body whose supporting hyperplane in a given direction $u$ has a distance $\sigma_{K}(u)$ from the origin; i.e., the support function of $\Pi K$ is $\sigma_{K}$. The Petty projection inequality $[\mathbf{1 9}$, p. 40] is

$$
I_{m}(\Pi K) V(K)^{n-1} \leq\left(\omega_{n} / \omega_{n-1}\right)^{n}
$$

with equality if and only if $K$ is an ellipsoid,

where $I_{m}(\Pi K)$ denotes the minimum of the volumes of the polar reciprocal bodies of $\Pi K$. Since $\Pi K$ is centrally symmetric, it follows (see, for example, $[13,19,20$, 23]) that

$$
I_{m}(\Pi K)=V\left(\Pi^{\circ} K\right)
$$

where $\Pi^{\circ} K$ denotes the polar reciprocal of $\Pi K$ with respect to (the unit sphere centered at) the origin. Since the boundary of $\Pi^{\circ} K$ can be represented in polar form by $r=\sigma_{K}(u)^{-1}$, the volume of $\Pi^{\circ} K$ is given by

$$
V\left(\Pi^{\circ} K\right)=\frac{1}{n} \int_{S^{n-1}} \sigma_{K}^{-n}(u) d S(u)
$$

It follows that the Petty projection inequality is the case $p=-n$ in Theorem 2 . The cases where $p>-n$, now, follow from the case $p=-n$ if we use (4). To see that the inequality (in Theorem 2) does not hold for $p<-n$, take $K$ to be any nonspherical ellipsoid, and use the case $p=-n$ in conjunction with (4).

We note that Theorem 2 completely solves the problem posed in [14]. We also note that for $n=2$ (the plane case) both theorems coincide.

As will be seen shortly, the case $p=-1$ in Theorem 2 is of particular interest. 
The projection measures (Quermassintegrale) $W_{0}, W_{1}, \ldots, W_{n}$ in $\mathbf{R}^{n}$ can be defined (see [9, p. 234]) by letting $W_{0}(K)=V(K), W_{n}(K)=\omega_{n}$, and, for $0<i<n$, letting

$$
\frac{\omega_{i}}{\omega_{n}} W_{n-i}(K)=\frac{\omega_{n-i}}{\omega_{n} c_{i n}} \int V_{i}\left(K \mid E_{i}\right) d \overline{\bar{E}}_{i}
$$

where all such integrals are to be taken over the entire space of freely rotating $i$-dimensional flats $E_{i}$ through the origin, $K \mid E_{i}$ denotes the projection of $K$ onto $E_{i}, V_{i}$ denotes $i$-dimensional volume and $d \bar{E}_{i}$ is the rotation density, normalized so that

where

$$
\int d \overline{\bar{E}}_{i}=\frac{\omega_{n} c_{i n}}{\omega_{n-i}}
$$

$$
c_{i n}=\left(\begin{array}{c}
n \\
i
\end{array}\right) \frac{\omega_{n-1} \cdots \omega_{n-i}}{\omega_{1} \cdots \omega_{i}} .
$$

The harmonic projection measures (harmonische Quermassintegrale) $\tilde{W}_{0}, \tilde{W}_{1}, \ldots$, $\tilde{W}_{n}$ in $\mathbf{R}^{n}$ are defined by Hadwiger $\left[9\right.$, p. 267] by letting $\tilde{W}_{0}(K)=V(K), W_{n}(K)=$ $\omega_{n}$, and, for $0<i<n$, letting

$$
\frac{\omega_{i}}{\omega_{n}} \tilde{W}_{n-i}(K)=\left[\frac{\omega_{n-i}}{\omega_{n} c_{i n}} \int V_{i}\left(K \mid E_{i}\right)^{-1} d \overline{\bar{E}}_{i}\right]^{-1} .
$$

It follows (see $[9$, p. 267]) that

$$
\tilde{W}_{i}(K) \leq W_{i}(K)
$$

with equality for $0<i<n$ if and only if the $(n-i)$-dimensional projections of $K$ have constant $(n-i)$-dimensional volume.

Obviously, we have

$$
W_{n-1}(K)=\omega_{n} M_{1}\left[b_{K} / 2\right] \text { and } \tilde{W}_{n-1}(K)=\omega_{n} M_{-1}\left[b_{K} / 2\right],
$$

while

$$
W_{1}(K)=\omega_{n} M_{1}\left[\sigma_{K} / \omega_{n-1}\right] \text { and } \tilde{W}_{1}(K)=\omega_{n} M_{-1}\left[\sigma_{K} / \omega_{n-1}\right] \text {. }
$$

The case $p=1$ in Theorem 1 is the Urysohn inequality,

$$
\omega_{n}^{n-1} V(K) \leq W_{n-1}(K)^{n}
$$

with equality if and only if $K$ is a ball,

while the case $p=-1$ in Theorem 1 is the stronger harmonic Urysohn inequality (see $[12,16])$,

$$
\omega_{n}^{n-1} V(K) \leq \tilde{W}_{n-1}(K)^{n},
$$

with equality if and only if $K$ is a ball.

Similarly, the case $p=1$ in Theorem 2 is the isepiphanic inequality,

$$
\omega_{n} V(K)^{n-1} \leq W_{1}(K)^{n},
$$

with equality if and only if $K$ is a ball,

while the case $p=-1$ in Theorem 2 is the stronger harmonic isepiphanic inequality,

$$
\omega_{n} V(K)^{n-1} \leq \tilde{W}_{1}(K)^{n},
$$

with equality if and only if $K$ is a ball. 
This last inequality is conjectured in [16, p. 147].

In light of the critical role played by the case $p=-n$ in both theorems, one is led to define affine projection measures $\Phi_{0}, \Phi_{1}, \ldots, \Phi_{n}$ in $\mathbf{R}^{n}$ by taking $\Phi_{0}(A)=$ $V(A), \Phi_{n}(A)=\omega_{n}$, and, for $0<i<n$, letting

Obviously, we have

$$
\frac{\omega_{i}}{\omega_{n}} \Phi_{n-i}(K)=\left[\frac{\omega_{n-i}}{\omega_{n} c_{i n}} \int V_{i}\left(K \mid E_{i}\right)^{-n} d \overline{\bar{E}}_{i}\right]^{-1 / n} .
$$

$$
\Phi_{i}(K) \leq \tilde{W}_{i}(K) \leq W_{i}(K)
$$

with equality for $0<i<n$ if and only if the $(n-i)$-dimensional projections of $K$ have constant $(n-i)$-dimensional volume.

As noted by Hadwiger [9, p. 267], the harmonic projection measure $\tilde{W}_{i}$ (viewed as a functional on the space of convex bodies in $\mathbf{R}^{n}$, endowed with the topology induced by the Hausdorff metric [9, p. 151]) is positive, continuous, bounded, monotone (increasing), homogeneous of degree $n-i$, and invariant under motions (translations and rotations). It is easy to verify that the affine projection measure $\Phi_{i}$ has exactly the same properties. has

Hadwiger [9, p. 268] proves that for the Minkowski (vector) sum $K_{1}+K_{2}$ one

$$
\tilde{W}_{i}\left(K_{1}+K_{2}\right)^{1 /(n-i)} \geq \tilde{W}_{i}\left(K_{1}\right)^{1 /(n-i)}+\tilde{W}_{i}\left(K_{2}\right)^{1 /(n-i)}
$$

i.e., $\tilde{W}_{i}^{1 /(n-i)}$ is concave. Similarly, following in the same manner as Hadwiger, one has

$$
\Phi_{i}\left(K_{1}+K_{2}\right)^{1 /(n-i)} \geq \Phi_{i}\left(K_{1}\right)^{1 /(n-i)}+\Phi_{i}\left(K_{2}\right)^{1 /(n-i)} .
$$

In terms of affine projection measures, the case $p=-n$ in Theorem 1 may be viewed as the affine Bieberbach inequality,

$$
\omega_{n}^{n-1} V(K) \leq \Phi_{n-1}(K)^{n},
$$

with equality if and only if $K$ is an ellipsoid,

while the case $p=-n$ in Theorem 2 (the Petty projection inequality) may be viewed as the affine isepiphanic inequality,

$$
\omega_{n} V(K)^{n-1} \leq \Phi_{1}(K)^{n}
$$

\section{with equality if and only if $K$ is an ellipsoid.}

For a given convex body $K$ in $\mathbf{R}^{n}$ and a direction $u \in S^{n-1}$ let $l_{K}(u, x)$ denote the length of the cord of $K$ that is orthogonal to $E_{u}$ and (when extended) passes through the point $x \in E_{u}$. Let $l_{K}(u)$ denote the mean length of chords of $K$ that are in the direction $u$; i.e.,

$$
l_{K}(u)=\frac{1}{\sigma_{K}(u)} \int_{K \mid E_{u}} l_{K}(u, x) d V_{n-1}(x),
$$

where $d V_{n-1}(x)$ is the $(n-1)$-dimensional volume element on $E_{u}$ at $x$. Clearly,

$$
l_{K}(u)=V(K) \sigma_{K}(u)^{-1} .
$$

The following 'dual' of the Urysohn inequality was conjectured by the author at the 1978 Oberwolfach 'Konvexe Körper' conference (see [27, p. 265]):

$$
\begin{gathered}
\frac{\omega_{n-1}}{n \omega_{n}} \int_{S^{n-1}} l_{K}(u) d S(u) \leq \omega_{n}^{(n-1) / n} V(K)^{1 / n}, \\
\text { with equality if and only if } K \text { is a ball. }
\end{gathered}
$$


This is an immediate consequence of the harmonic isepiphanic inequality (case $p=$ -1 in Theorem 2) if we use (5).

The inequality in Theorem 2 with $p=-n$ (the Petty projection inequality) can be used to obtain brightness-volume inequalities analogous to some width-volume inequalities obtained by Chakerian [6, 7], Chakerian and Sangwine-Yager [8], and Lutwak [15].

For convex bodies $K_{1}, \ldots, K_{n}$ in $\mathbf{R}^{n}$, and for a real number $p \neq 0$, we can define $S_{p}\left(K_{1}, \ldots, K_{n}\right)$ by:

$$
S_{p}\left(K_{1}, \ldots, K_{n}\right)=\left[\frac{1}{n \omega_{n}} \int_{S^{n-1}}\left[\sigma_{K_{1}}(u) \cdots \sigma_{K_{n}}(u)\right]^{p} d S(u)\right]^{1 / p} .
$$

Following as in [15], we can use the Petty projection inequality and the Hölder inequality $[10$, p. 140$]$ to obtain

$$
\begin{aligned}
& \left(\omega_{n-1}^{n} / \omega_{n}^{n-1}\right)\left[V\left(K_{1}\right) \cdots V\left(K_{n}\right)\right]^{(n-1) / n} \leq S_{-1}\left(K_{1}, \ldots, K_{n}\right), \\
& \text { with equality if and only if the } K_{i} \text { are homothetic ellipsoids. }
\end{aligned}
$$

The inequality (6) is a strengthened form of

$$
\begin{gathered}
\left(\omega_{n-1}^{n} / \omega_{n}^{n-1}\right)\left[V\left(K_{1}\right) \cdots V\left(K_{n}\right)\right]^{(n-1) / n} \leq S_{1}\left(K_{1}, \ldots, K_{n}\right), \\
\text { with equality if and only if all } K_{i} \text { are balls. }
\end{gathered}
$$

In connection with the last two inequalities, we note that a centrally symmetric body $K$ always has volume greater than that of any other convex body whose brightness function is the same as that of $K$ (see [18 and 24]).

If we combine (5) and (6) we obtain an inequality in the spirit of the concurrent cross-section inequality of Busemann [4] (also see [17]):

$$
\begin{aligned}
& \frac{1}{n} \int_{S^{n-1}} l_{K_{1}}(u) \cdots l_{K_{n}}(u) d S(u) \leq\left(\omega_{n}^{n} / \omega_{n-1}^{n}\right)\left[V\left(K_{1}\right) \cdots V\left(K_{n}\right)\right]^{1 / n}, \\
& \text { with equality if and only if the } K_{i} \text { are homothetic ellipsoids. }
\end{aligned}
$$

For a convex body $K$ and a direction $u \in S^{n-1}$, let $V_{K}(u)$ denote the volume of the right cylinder circumscribed about $K$ whose generators are orthogonal to $u$. Clearly $V_{K}(u)=b_{K}(u) \sigma_{K}(u)$. By using the inequalities of Theorems 1 and 2 in conjunction with the Hölder inequality we obtain

$$
\begin{aligned}
& \frac{2 \omega_{n-1}}{\omega_{n}} V(K) \leq\left[\frac{1}{n \omega_{n}} \int_{S^{n-1}} V_{K}(u)^{-1} d S(u)\right]^{-1}, \\
& \text { with equality if and only if } K \text { is a ball. }
\end{aligned}
$$

This is a strengthened form of the inequality

$$
\begin{aligned}
& \frac{2 \omega_{n-1}}{\omega_{n}} V(K) \leq \frac{1}{n \omega_{n}} \int_{S^{n-1}} V_{K}(u) d S(u), \\
& \text { with equality if and only if } K \text { is a ball, }
\end{aligned}
$$

which was obtained by Knothe [11] for $n=3$ and proved by Chakerian [5] for $n \geq 3$.

The author would like to thank Professors R. Schneider and K. Leichtweiss for several informative observations regarding the Blaschke-Santaló inequality. The author would also like to thank the referee for reference [22]. 


\section{REFERENCES}

1. W. Blaschke, Über affine Geometrie VII: Neue Extremeigenschaften von Ellipse und Ellipsoid, Leipziger Berichte. 69 (1917), 306-318.

2. E. D. Bolker, A class of convex bodies, Trans. Amer. Math. Soc. 145 (1969), 323-345.

3. T. Bonnesen and W. Fenchel, Theorie der konvexen Körper, Springer-Verlag, Berlin, 1934.

4. H. Busemann, Volume in terms of concurrent cross-sections, Pacific J. Math. 3 (1953), 1-12.

5. G. D. Chakerian, The mean volume of boxes and cylinders circumscribed about a convex body, Israel J. Math. 12 (1972), 249-256.

6. __ Isoperimetric inequalities for the mean width of a convex body, Geom. Dedicata 1 (1973), 356-362.

7.

8. G. D. Chakerian and J. R. Sangwine-Yager, A generalization of Minkowski's inequality for plane convex sets, Geom. Dedicata 8 (1979), 437-444.

9. H. Hadwiger, Vorlesungen über Inhalt, Oberfläche, und Isoperimetrie, Springer-Verlag, Berlin, 1957.

10. G. H. Hardy, J. E. Littlewood and G. Pólya, Inequalities, Cambridge Univ. Press, Cambridge, 1934.

11. H. Knothe, Inversion of two theorems of Archimedes, Michigan Math. J. 4 (1957), 53-56.

12. E. Lutwak, A general Bieberbach inequality, Math. Proc. Cambridge Philos. Soc. 78 (1975), 493-496.

13. __ Width-integrals of convex bodies, Proc. Amer. Math. Soc. 53 (1975), 435-439.

14. __ A dual of the isepiphanic inequality, Arch. Math. 27 (1976), 206-208.

15. _ - Mixed width-integrals of convex bodies, Israel J. Math. 28 (1977), 249-253.

16. __ Mean dual and harmonic cross-sectional measures, Ann. Mat. Pura Appl. 119 (1979), 139-148.

17. C. M. Petty, Centroid surfaces, Pacific J. Math. 11 (1961), 1535-1547.

18. __ Projection bodies, (Proc. Colloq. Convexity, Copenhagen, 1965), Kobenhavns Univ. Mat. Inst., Copenhagen, 1967, pp. 234-241.

19. __ Isoperimetric problems, (Proc. Conf. Convexity and Combinatorial Geometry, Univ. of Oklahoma, June 1971), Univ. of Oklahoma, Norman, 1971, pp. 26-41.

20. __ Geominimal surface area, Geom. Dedicata 3 (1974), 77-97.

21. __ Affine isoperimetric problems, Discrete Geometry and Convexity (Eds., J. E. Goodman, et al.) New York Acad. Sci. (to appear).

22. J. Saint Raymond, Sur le volume des corps convexes symétriques, Séminaire Choquet, 20e année (1980/81), Initiation à l'analyse, Comm. No. 11, 25 pp., Secrétariat Math., Paris, 1981.

23. L. A. Santaló, Un invariante afíne para los cuerpos convexos del espacio de $n$ dimensiones, Portugal. Math. 8 (1949), 155-161.

24. R. Schneider, $Z u$ einem problem von Shepard über die Projektionen konvexer Körper, Math. Z. 101 (1967), 71-82.

25. R. Schneider, Random polytopes generated by anisotropic hyperplanes, Bull. London Math. Soc. 14 (1982), 549-553.

26. R. Schneider and W. Weil, Zonoids and related topics, Convexity and Its Applications (P. M. Gruber and J. M. Wills, Eds.), Birkhäuser, Basel, 1983, pp. 296-317.

27. J. Tölke and J. M. Wills, Contributions to geometry, Birkhäuser, Basel, 1979.

Department of Mathematics, Polytechnic Institute of New York, Brooklyn, NEW YORK 11201 